\title{
Implementation of Bayesian methods to identify SNP and haplotype regions with transmission ratio distortion across the whole genome: TRDscan v.1.0
}

\author{
S. Id-Lahoucine, ${ }^{1,2 *}$ A. Cánovas, ${ }^{1}$ C. Jaton, ${ }^{1,3}$ F. Miglior, ${ }^{1,4}$ P. A. S. Fonseca, ${ }^{1,5}$ M. Sargolzaei, ${ }^{1,6}$ S. Miller, ${ }^{1,7}$ \\ F. S. Schenkel, ${ }^{1}$ J. F. Medrano, ${ }^{8}$ and J. Casellas ${ }^{2}$ \\ ${ }^{1}$ Centre for Genetic Improvement of Livestock, Department of Animal Biosciences, University of Guelph, Guelph N1G 2W1, Ontario, Canada \\ ${ }^{2}$ Departament de Ciència Animal i dels Aliments, Universitat Autònoma de Barcelona, Bellaterra 08193, Barcelona, Spain \\ ${ }^{3}$ The Semex Alliance, Guelph N1G 3Z2, Ontario, Canada \\ ${ }^{4}$ Canadian Dairy Network, Guelph N1K 1E5, Ontario, Canada \\ ${ }^{5}$ Departamento de Biologia Geral, Universidade Federal de Minas Gerais, Belo Horizonte 31270-901, Brazil \\ ${ }^{6}$ HiggsGene Solutions Inc., Guelph N1G 4S7, Ontario, Canada \\ ${ }^{7}$ Angus Genetics Inc., St. Joseph, MO 64506 \\ ${ }^{8}$ Department of Animal Science, University of California-Davis, Davis 95616
}

\section{ABSTRACT}

Realized deviations from the expected Mendelian inheritance of alleles from heterozygous parents have been previously reported in a broad range of organisms (i.e., transmission ratio distortion; TRD). Various biological mechanisms affecting gametes, embryos, fetuses, or even postnatal offspring can produce patterns of TRD. However, knowledge about its prevalence and potential causes in livestock species is still scarce. Specific Bayesian models have been recently developed for the analyses of TRD for biallelic loci, which accommodated a wide range of population structures, enabling TRD investigation in livestock populations. The parameterization of these models is flexible and allows the study of overall (parent-unspecific) TRD and sire- and dam-specific TRD. This research aimed at deriving Bayesian models for fitting TRD on the basis of haplotypes, testing the models for both haplotypeand SNP-based methods in simulated data and actual Holstein genotypes, and developing a specific software for TRD analyses. Results obtained on simulated data sets showed that the statistical power of the analysis increased with sample size of trios (n), proportion of heterozygous parents, and the magnitude of the TRD. On the other hand, the statistical power to detect TRD decreased with the number of alleles at each loci. Bayesian analyses showed a strong Pearson correlation coefficient $(\geq 0.97)$ between simulated and estimated TRD that reached the significance level of Bayes factor $\geq 10$ for both single-marker and haplotype analyses when $\mathrm{n} \geq 25$. Moreover, the accuracy in terms of the mean absolute error decreased with the increase of the

Received June 28, 2018.

Accepted December 8, 2018.

*Corresponding author: sidlahou@uoguelph.ca sample size and increased with the number of alleles at each loci. Using real data (55,732 genotypes of Holstein trios), SNP- and haplotype-based distortions were detected with overall TRD, sire-TRD, or dam-TRD, showing different magnitudes of TRD and statistical relevance. Additionally, the haplotype-based method showed more ability to capture TRD compared with individual SNP. To discard possible random TRD in real data, an approximate empirical null distribution of TRD was developed. The program TRDscan v.1.0 was written in Fortran 2008 language and provides a powerful statistical tool to scan for TRD regions across the whole genome. This developed program is freely available at http://www.casellas.info/files/TRDscan.zip.

Key words: transmission ratio distortion, heterozygous parent, haplotype, Bayesian model

\section{INTRODUCTION}

Deviations from the expected Mendelian inheritance of alleles from heterozygous parents at a locus have been reported in a broad range of organisms (e.g., Evans et al., 1994; Hall and Willis, 2005; Axelsson et al., 2010; Casellas et al., 2012). This phenomenon is well known as transmission ratio distortion (TRD; Silver, 1993; Pardo-Manuel de Villena et al., 2000) and can be viewed as the consequence of multiple biological mechanisms, including meiotic drive (Agulnik et al., 1990; Lyon, 1991; Dyer et al., 2007), embryo or fetal failure (Wakasugi, 1974), germline selection (Hastings, 1991), gametic competition (Zöllner et al., 2004), imprint resetting error (Naumova et al., 2001; Yang et al., 2008), and differential postnatal viability (Moore, 2006; Casellas, 2007). However, the non-Mendelian segregation remains ambiguous (Nadeau, 2017) and current knowledge about its prevalence and possible causes in livestock species is still scarce. The link to impaired fer- 
tility (Silver, 1989) and differential offspring survival at different stages (Dean et al., 2006; Eversley et al., 2010) emphasizes the relevance of TRD, which could be an outstanding alternative to search for genomic regions that may contain potential causative mutations directly affecting reproductive traits, as illustrated by the success of previous methods targeting a particular type of TRD based on the absence of homozygous haplotypes (e.g., VanRaden et al., 2011; Hoff et al., 2017).

Current availability of large amounts of genomic data including genotyped parents and unselected genotyped offspring (within families) enables the study of TRD in the whole genome. Recently, Casellas et al. (2014) developed Bayesian models for the analyses of TRD, providing a useful methodology applicable to livestock populations even for pedigrees with incomplete trios. However, these models are limited to biallelic loci. From a genetic inheritance perspective and taking into account that large haplotypes are potentially transmitted from parents to offspring for several generations, the implementation of TRD analyses using haplotypes can be a more powerful and precise method to unravel the genomic regions under TRD. The objectives of this research were to introduce Bayesian models of TRD for haplotypes, to evaluate the statistical power and accuracy for both SNP- and haplotype-based methods with simulated data and to test the models in genotypes of Holstein cattle. The final goal was to develop and provide a software as a statistical tool to scan for TRD regions across the whole genome.

\section{MATERIALS AND METHODS}

\section{TRD Models for Biallelic Loci (SNP-Based Method)}

Casellas et al. (2014) developed Bayesian models of TRD analyses for biallelic loci focusing on (1) the overall (parent-unspecific) TRD or (2) specific paternal- and maternal-origin TRD (sire- and dam-specific TRD). These 2 models parameterize the probability of inheritance $(\mathrm{P})$ of a given allele from a heterozygous parent (A/B) by including (1) one overall TRD effect $(\alpha)$ or $(2)$ both sire- $\left(\alpha_{s}\right)$ and dam-specific TRD effects $\left(\alpha_{d}\right)$ :

$$
\begin{gathered}
\mathrm{P}(\mathrm{A})=1-\mathrm{P}(\mathrm{B})=0.5+\alpha \text { and } \mathrm{P}(\mathrm{B})= \\
1-\mathrm{P}(\mathrm{A})=0.5-\alpha \\
\mathrm{P}_{\mathrm{i}}(\mathrm{A})=1-\mathrm{P}_{\mathrm{i}}(\mathrm{B})=0.5+\alpha_{\mathrm{i}} \text { and } \mathrm{P}_{\mathrm{i}}(\mathrm{B})= \\
1-\mathrm{P}_{\mathrm{i}}(\mathrm{A})=0.5-\alpha_{\mathrm{i}}
\end{gathered}
$$

with $\mathrm{i}=[\mathrm{s} \mathrm{U} d]$, where $\alpha, \alpha_{\mathrm{s}}$, and $\alpha_{\mathrm{d}}$ are TRD parameters that assumed flat priors within a parametric space ranging from -0.5 to 0.5 . Homozygous parents do not contribute relevant information to the analysis; thereby, the probabilities of transmission reduce to 0 or 1. Under a Bayesian implementation, the conditional posterior probabilities of the TRD parameters are defined as

$$
\begin{gathered}
\mathrm{p}(\alpha \mid \mathbf{y}) \propto \mathrm{p}(\mathbf{y} \mid \alpha) \mathrm{p}(\alpha) \text { and } \\
\mathrm{p}\left(\alpha_{\mathrm{s}}, \alpha_{\mathrm{d}} \mid \mathbf{y}\right) \propto \mathrm{p}\left(\mathbf{y} \mid \alpha_{\mathrm{s}}, \alpha_{\mathrm{d}}\right) \mathrm{p}\left(\alpha_{\mathrm{s}}\right) \mathrm{p}\left(\alpha_{\mathrm{d}}\right),
\end{gathered}
$$

where $\mathbf{y}$ is the column vector of genotypes of the offspring generation.

\section{Derivation of TRD Models for Multiallelic Loci (Haplotype-Based Method)}

The previous model was developed for biallelic loci. An extension of TRD models to account for haplotype regions or microsatellites markers, with more than 2 alleles (multiallelic), could be of special relevance for both research and the livestock industry. To capture all the possible interaction between alleles, the same parameterization can be assumed, but estimating different TRD for each heterozygous pairwise combination of 2 alleles ( $\mathrm{j}$ and $\mathrm{k}$ ). The Bayesian development of TRD models for multiallelic loci was

$$
\begin{gathered}
\mathrm{p}\left(\alpha_{12}, \alpha_{13}, \ldots \alpha_{(\mathrm{n}-1) \mathrm{n}} \mid \mathbf{y}\right) \propto \mathrm{p}\left(\mathbf{y} \mid\left(\alpha_{12}, \alpha_{13}, \ldots\right.\right. \\
\left.\alpha_{(\mathrm{n}-1) \mathrm{n}}\right) \mathrm{p}\left(\alpha_{12}\right) \mathrm{p}\left(\alpha_{13}\right) \ldots \mathrm{p}\left(\alpha_{(\mathrm{n}-1) \mathrm{n}}\right) \text { and } \\
\mathrm{p}\left(\alpha_{\mathrm{s} 12}, \alpha_{\mathrm{d} 12}, \alpha_{\mathrm{s} 13}, \alpha_{\mathrm{d} 13}, \ldots \alpha_{\mathrm{s}(\mathrm{n}-1) \mathrm{n}}, \alpha_{\mathrm{d}(\mathrm{n}-1) \mathrm{n}} \mid \mathbf{y}\right) \\
\propto \mathrm{p}\left(\mathbf{y} \mid\left(\alpha_{\mathrm{s} 12}, \alpha_{\mathrm{d} 12}, \alpha_{\mathrm{s} 13}, \alpha_{\mathrm{d} 13}, \ldots \alpha_{\mathrm{s}(\mathrm{n}-1) \mathrm{n}}, \alpha_{\mathrm{d}(\mathrm{n}-1) \mathrm{n}}\right) \mathrm{p}\left(\alpha_{\mathrm{s} 12}\right)\right. \\
\mathrm{p}\left(\alpha_{\mathrm{d} 12}\right) \mathrm{p}\left(\alpha_{\mathrm{s} 13}\right) \mathrm{p}\left(\alpha_{\mathrm{d} 13}\right) \ldots \mathrm{p}\left(\alpha_{\mathrm{s}(\mathrm{n}-1) \mathrm{n}}\right) \mathrm{p}\left(\alpha_{\mathrm{d}(\mathrm{n}-1) \mathrm{n}}\right),
\end{gathered}
$$

where $\mathrm{n}$ is the number of alleles for the specific loci, $\mathbf{y}$ is the vector of offspring's genotypes, and $\alpha_{\mathrm{jk}}, \alpha_{\mathrm{sjk}}$, and $\alpha_{\mathrm{djk}}$ are the overall and sire- and dam-specific TRD parameters for the specific heterozygous combination of alleles $\mathrm{j}$ and $\mathrm{k}$, respectively.

When some genotypes in the parental generation (incomplete trios) are missing, an extension of Casellas et al. (2014) model can also be adapted within the context of the haplotype-based approach (see Appendix). On the other hand, to estimate TRD parameters for a specific allele independent of the combined allele, a second procedure of estimating TRD for haplotypes analyses can be applied. Hence, the biallelic haplotype procedure could be based on remodeling the multiallelic loci to a biallelic loci regarding a particular haplotype allele and by assigning the remaining alleles as the opposite one (i.e., the chosen haplotype allele for the analysis plus 
all other alleles amalgamated in a single allele). Within this context, by assuming the same parameterization and independent interaction between alleles, TRD parameters for each particular allele can be estimated with more statistical power. One or both of haplotype analyses (heterozygous pairwise combination and biallelic haplotype procedures) may be used according to the objectives, limitations, and assumptions of a study.

\section{Likelihood}

The likelihood of data consists of a straightforward multiplication of the corresponding probabilities for each offspring as

$$
\begin{aligned}
& \mathrm{p}(\mathbf{y} \mid \alpha)=\prod_{\mathrm{n}} \mathrm{P}_{\text {off }}\left(\mathrm{y}_{\mathrm{i}}\right) \text { and } \\
& \mathrm{p}\left(\mathbf{y} \mid \alpha_{\mathrm{s}}, \alpha_{\mathrm{d}}\right)=\prod_{\mathrm{n}} \mathrm{P}_{\text {off }}\left(\mathrm{y}_{\mathrm{i}}\right),
\end{aligned}
$$

where $\mathrm{n}$ is the total number of offspring and $\mathrm{P}_{\text {off }}$ and $y_{\mathrm{i}}$ are the probability and the genotype of the ith offspring, respectively. The probability of the genotype of each offspring was defined by parents' genotypes and TRD parameters. Considering, for example, a heterozygous offspring from a heterozygous-by-heterozygous mating, the probability including TRD effects is

$$
\begin{gathered}
\mathrm{P}_{\text {off }}(\mathrm{AB})=[(0.5+\alpha)(0.5-\alpha)] \\
+[(0.5-\alpha)(0.5+\alpha)] \text { and } \\
\mathrm{P}_{\text {off }}(\mathrm{AB})=\left[\left(0.5+\alpha_{\mathrm{s}}\right)\left(0.5-\alpha_{\mathrm{d}}\right)\right] \\
+\left[\left(0.5-\alpha_{\mathrm{s}}\right)\left(0.5+\alpha_{\mathrm{d}}\right)\right] .
\end{gathered}
$$

For SNP-by-SNP and biallelic haplotype procedure analyses, more efficient analyses can be performed by using a multinomial process involving all types of informative matings on parental generation (e.g., $\mathrm{AB} \times \mathrm{AA}$, $\mathrm{AB} \times \mathrm{BB}, \mathrm{AA} \times \mathrm{AB}, \mathrm{BB} \times \mathrm{AB}$, and $\mathrm{AB} \times \mathrm{AB})$. Under this simplification and assuming one overall TRD, the likelihood of the data becomes

$$
\begin{aligned}
& \mathrm{p}(\mathbf{y} \mid \alpha) \propto \prod_{\mathrm{i}=1}^{3} \frac{\mathrm{n}_{\mathrm{i}} !}{\mathrm{n}_{\mathrm{AA}, \mathrm{i}} ! \mathrm{n}_{\mathrm{AB}, \mathrm{i}} ! \mathrm{n}_{\mathrm{BB}, \mathrm{i}} !} \times \mathrm{P}_{\text {off }, \mathrm{i}}(\mathrm{AA})^{\mathrm{n}_{\mathrm{AA}, \mathrm{i}}} \\
& \times \mathrm{P}_{\mathrm{off}, \mathrm{i}}(\mathrm{AB})^{\mathrm{n}_{\mathrm{AB}, \mathrm{i}}} \times \mathrm{P}_{\text {off }, \mathrm{i}}(\mathrm{BB})^{\mathrm{n}_{\mathrm{BB}, \mathrm{i}}} .
\end{aligned}
$$

where $\mathrm{P}_{\text {off,i }}$ is the probability of an offspring genotype from the ith mating; $\mathrm{n}_{\mathrm{AA}, \mathrm{i}}, \mathrm{n}_{\mathrm{AB}, \mathrm{i}}$, and $\mathrm{n}_{\mathrm{BB}, \mathrm{i}}$ are the number of $\mathrm{AA}, \mathrm{AB}$, and $\mathrm{BB}$ offspring genotypes from the specific ith mating, respectively. $n_{i}$ is the sum of $n_{A A, i}$,
$n_{A B, i}$, and $n_{B B, i}$ offspring genotypes. When assuming a parent-specific TRD, 5 kinds of matings were differentiated in the multinomial expression (i.e., $\prod_{i=1}^{5}$ ).

\section{Inference of TRD Parameters and Bayes Factor Via Markov Chain Monte Carlo Sampling}

A uniform proposal distribution between -0.5 and 0.5 was used for the purpose of sampling. Mean and standard deviation of TRD effects were obtained via a Markov chain Monte Carlo method using MetropolisHastings algorithm (Hastings, 1970).

The statistical relevance of each TRD parameter was tested by a Bayes factor (BF) approach (Kass and Raftery, 1995), which is a ratio of probabilities between the model with TRD and a virtual model with null TRD. Following García-Cortés et al. (2001) and Varona et al. (2001), the BF considering one overall TRD was obtained as

$$
\mathrm{BF}_{\alpha}=\frac{\mathrm{p}(\alpha=0)}{\mathrm{p}(\alpha=0 \mid \mathrm{y})}
$$

When more than one single parameter were included in the model, the same approach was used to compute the $\mathrm{BF}$ for each parameter. The marginal posterior density of each parameter was obtained by averaging the full conditional densities of each Markov chain Monte Carlo iteration at $\alpha_{i}=0$ (or $\left.\alpha_{j k}=0\right)$ applying the RaoBlackwell argument (Gelfand and Smith, 1990). The final $\mathrm{BF}$ for each parameter was calculated as a mean of the BF estimates obtained across iterations (with a lag interval of 10 iterations).

\section{Validation of the TRD Models Using Simulated Data}

For biallelic loci, parental generations were stochastically simulated under 2 strategies: (1) sampling a random allele frequency between 0.05 and 0.95 for both parents and (2) assuming dam's genotypes based on a random frequency of alleles, whereas the sires were heterozygous. The pedigree structures were simulated with 3 different sex ratios (sire:dam): (1) parent-offspring trios designed with a sex ratio of 1:1, (2) structure similar to the beef industry with a sex ratio of 1:25 (Boyd, 1993), and (3) structure similar to the dairy industry with a sex ratio of 1:100 (Sun et al., 2013). Different sample sizes of trios (n) ranging between 25 and 500 were generated under a random mating and considering one offspring per dam. The genotypes of offspring generation were simulated according to Mendelian inheritance model, but they included the TRD effects for heterozygotes parents as described previously (i.e., 
the first and second alleles were inherited with probabilities of $0.5+\alpha$ and $0.5-\alpha$, respectively). Different values of TRD, sampled from a uniform distribution ranging from -0.5 to 0.5 , were generated across 10,000 replicates for each simulation scenario.

For the multiallelic loci analyses, several loci with 4, 10, and 20 alleles at loci were considered. Pedigrees of 500 and 1,250 trios were generated with a sex ratio of 1:25 (20 and 50 sires, respectively). Random genotypes were assigned to the parental generation. Offspring generations were simulated based on parents' genotypes and under different scenarios of TRD, where it was applied in 1,2, or 4 alleles at loci.

\section{Validation of the TRD Models Using Real Data}

Genotypes from North-American Holstein dairy cattle were provided by Canadian Dairy Network (Guelph, ON). A total of 64,996 genotyped animals, including 2,031 sires, 13,708 dams, and 55,732 parent-offspring (trios), were included in the analyses. Animals were genotyped with different SNP genotyping arrays: 40,935 with the BovineSNP50 BeadChip (55,647 SNP; Illumina Inc., San Diego, CA), 15,719 with the GeneSeek Genomic Profiler-HD (77,068 SNP; Gene Seek, Lincoln, NE), 5,775 with the GGP Bovine 150K Array $(139,914$ SNP; Gene Seek), 2,185 with the Zoetis Medium Density Version 2 (60,914 SNP; Zoetis, Florham Park, NJ), and 382 with The BovineHD BeadChip (777,962 SNP; Illumina Inc.). A total of 44,369 autosomal SNP used usually for genomic evaluations by the Canadian Dairy Network were selected to test TRD models.

\section{Statistical Analyses}

The data were phased and missing SNP were imputed using FImpute (Sargolzaei et al., 2014). The TRD was evaluated across the 44,369 autosomal SNP using SNP-by-SNP and haplotype analyses. Haplotype sliding windows of $2,4,7,10$, and $20 \mathrm{SNP}$ were tested. For SNP-by-SNP analysis, both imputed and nonimputed data were tested with the purpose of assessing possible mitigation of the effect of genotyping error on TRD estimation. For the analyses several Markov chain Monte Carlo with $11,000,55,000$, or 110,000 iterations were tested to estimate TRD parameters; the first $1,000,5,000$, or 10,000 iterations were discarded as burn-in, respectively. The accuracy of the models was evaluated by the mean absolute error (MAE) and Pearson correlation coefficients $(\boldsymbol{\rho})$ between simulated and estimated TRD. The convergence of the models was checked by visual inspection and the effective chain size was obtained for each sample using Geyer's (1992) method.

\section{RESULTS AND DISCUSSION}

\section{Evaluation of TRD Models on Simulated Data}

Statistical Power. The BF is the standard Bayesian tool to quantify the support for a model over another using the posterior probability of the 2 competing models (Kass and Raftery, 1995). In TRD analyses, BF measures the change in the odds in favor of the model including TRD relative to the other model with null TRD $(\alpha=0)$, testing the statistical relevance of the full model. Considering the case of the biallelic loci as an example, the results showed higher BF when the magnitude of TRD increased (Figure 1), that is, displaying more statistical evidence in favor of the full model as TRD magnitude increases, indicating strong $(\mathrm{BF} \geq 10)$ or even decisive evidence $(\mathrm{BF} \geq 100)$ according to the Jeffreys (1984) scale. When there was no TRD or it was small, the $\mathrm{BF}$ was low or extremely low $(\mathrm{BF}<1)$, supporting the model without TRD. In addition, the $\mathrm{BF}$ also increased with larger sample sizes, allowing moderate and low $|\mathrm{TRD}|(\sim 0.05)$ to be detected with more power (Figure 1 ). The same results were obtained for the multiallelic loci. The BF can be viewed as a measure of the importance of the TRD detected given its ability to combine both magnitude of TRD and sample size of trios simultaneously.

The statistical power to detect overall TRD was clearly affected by the proportion of heterozygous sires and dams (Figure 1). Higher proportions of heterozygous parents resulted in higher $\mathrm{BF}$, because of the increase of the number of informative parents, therefore evidencing the TRD. For parent-specific TRD, the statistical power increased with higher proportions of heterozygous sires for sire-TRD and higher proportions of heterozygous dams for dam-TRD (results not shown). On the other hand, the sex ratio did not directly influence the statistical power. However, it determined the number of sires, and consequently, the available genetic variability and the proportion of heterozygous sires. Therefore, more statistical power was obtained when most of the sires were heterozygous, a situation that was observed more frequently when few males monopolized reproduction in the population (i.e., sex ratio $=$ $1: 100)$.

The factors that determine the statistical power (i.e., sample size and proportion of heterozygous parents) can be combined in one single factor, the number of informative offspring. This can be the number of offspring from one or both heterozygous parents for the overall TRD and from heterozygous sires and dams for sire- and dam-TRD, respectively. Figure 2 shows the statistical power expected for the detection of overall TRD or sire- and dam-TRD according to the number 

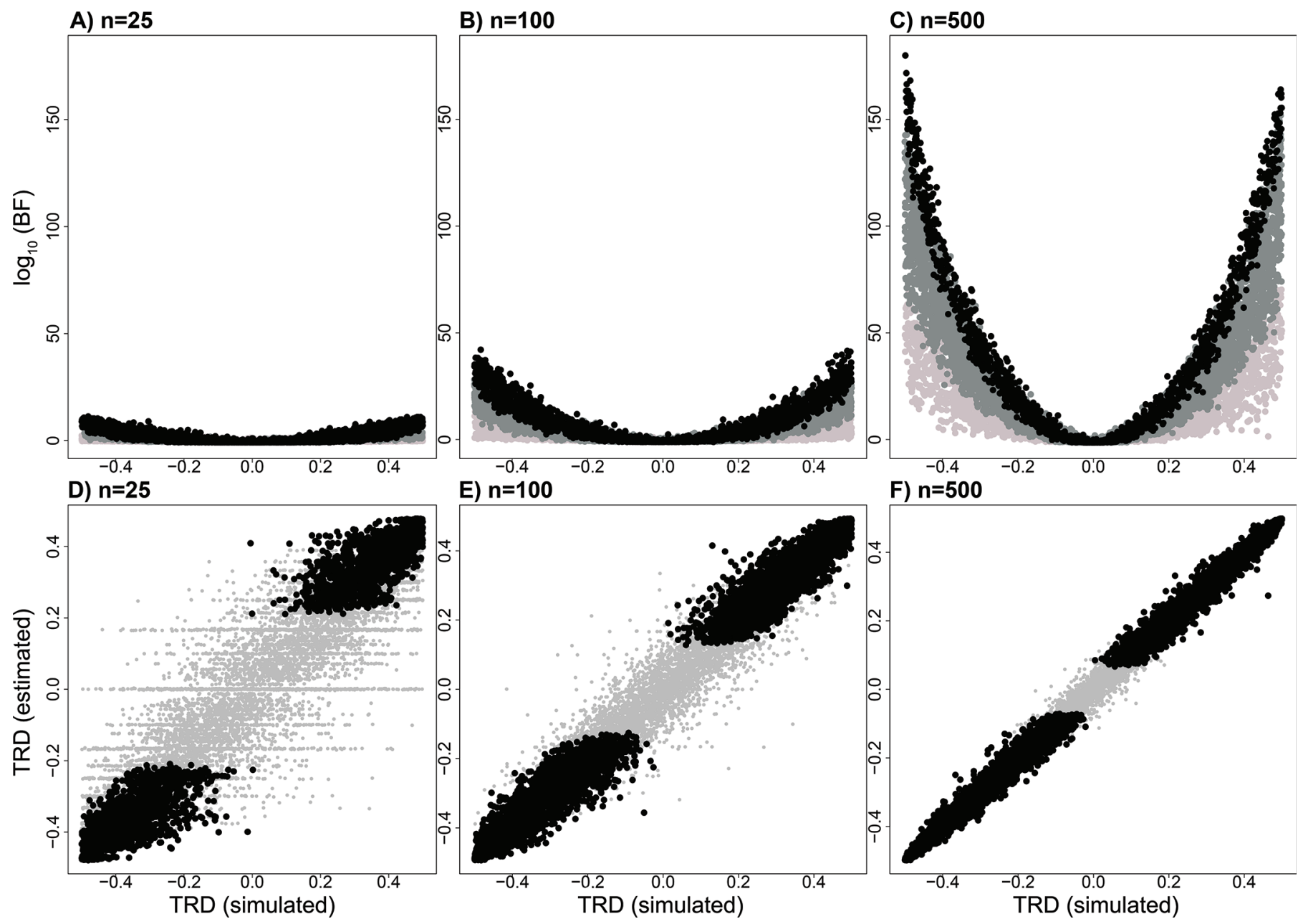

Figure 1. Bayes factor (BF) as a function of the magnitude of transmission ratio distortion (TRD) and average proportion of heterozygous parents ( $>0.5$ black, $0.25 \leq 0.5$ gray, $<0.25$ light gray; A-C) for different sample size of trios (n) with a sex ratio of 1:25; the simulated TRD plotted against estimated $\mathrm{TRD}$ ( $\mathrm{BF} \geq 10$ black, $\mathrm{BF}<10$ gray; $\mathrm{D}-\mathrm{F}$; biallelic loci).

of informative offspring. These results are applicable for both biallelic and multiallelic loci. Higher BF values were observed for the overall TRD than for parent-specific TRD. This is due to the fact that twice as many parameters are needed to estimate parent-specific TRD than the overall TRD.

Accuracy of Estimation. The MAE decreased with the increase in sample size of trios and, more specifically, the number of informative offspring (Tables 1 and 2), indicating higher estimation accuracy. In the same way, more quasi-perfect match between simulated and predicted values (i.e., high accuracy) was observed with larger sample sizes (Figure 1D-1F). In addition, the Bayesian analyses of TRD reached high accuracy in terms of Pearson correlation coefficients between simulated and estimated TRD $(\geq 0.97)$ when the BF clearly favored the model with TRD $(\mathrm{BF} \geq 10)$ when $\mathrm{n} \geq 25$. It should be noted that high $\rho$ was obtained also for small sample size $(\mathrm{n}=25)$, showing that even if higher MAE is observed with small $n$, the estimated TRD still persists highly correlated with the simulated TRD. Nevertheless, low estimation accuracy was observed when all TRD estimations were included, not only those with strong evidence. On the other hand, the dispersion of TRD parameters also supported the accuracy of the models. In this sense, small variances (or short credible interval) were obtained when relevant TRD were detected. More dispersion on TRD parameters was observed when the number of the informative offspring was very limited, as also evidenced by the observed MAE in small sample size, which usually were distortions discarded as random TRD (see the Approximate Empirical Null Distribution of TRD section for more details). Thus, whereas the standard deviation was $\sim 0.05$ for small size $(\mathrm{n}=25)$, it decreased to $\sim 0.01$ for $\mathrm{n}=500$ and even less with a larger sample size. 
a)

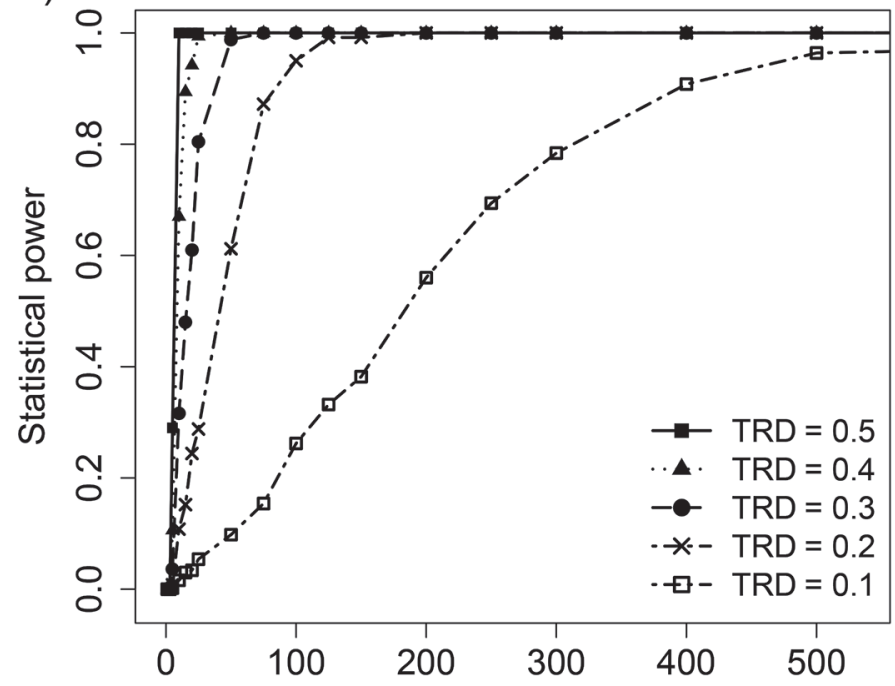

b)

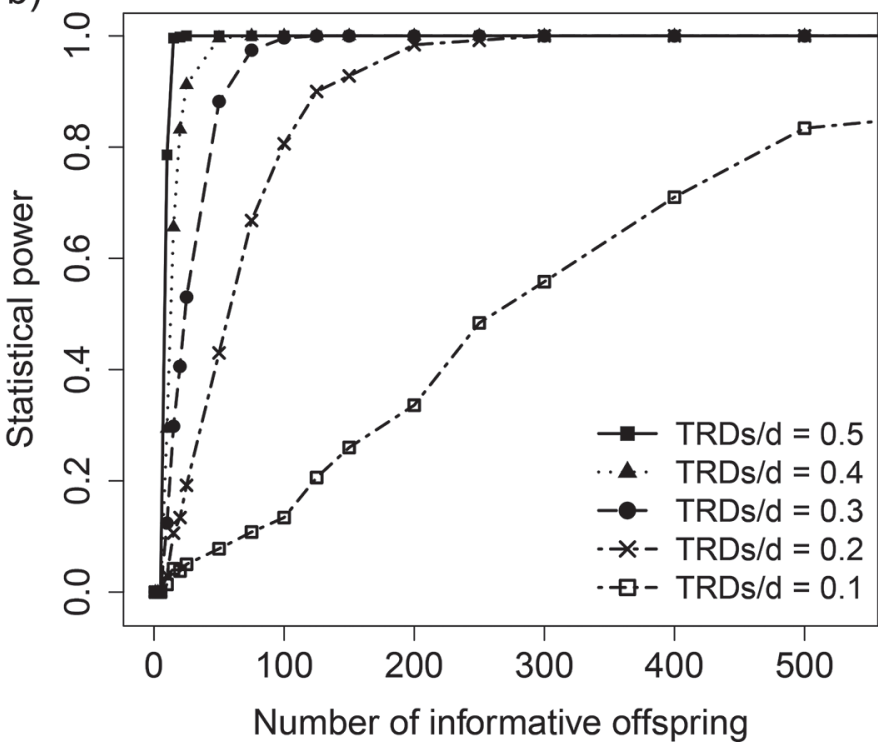

Figure 2. Statistical power to detect overall transmission ratio distortion (TRD) and sire- (s) or dam- (d) specific TRD with Bayes factor $\geq 10$ as a function of the number of informative offspring (biallelic loci). Informative offspring: offspring from one or both heterozygous parents for the overall TRD and from the specific heterozygous parent for the parent-specific TRD.

The standard deviation of the TRD parameters must be taken into account to obtain an approximate credible interval for TRD effects and to double check the convergence of the models.

Multiallelic Loci Analysis. The main differences in the analyses of TRD on multiallelic loci compared with on biallelic loci were a reduction in the $\mathrm{BF}$ and an increase in the MAE when the number of alleles increased for a given fixed sample size (Table 2). These were caused by the number of parameters estimated and the amount of data available for each heterozygous pairwise combination. It is worth to notice that all results for the biallelic loci should generalize to multiallelic loci given the similar parameterization. Within this context, similar accuracy was obtained for alleles detected with significant TRD $(\mathrm{BF} \geq 10)$. These results demonstrated the ability to detect haplotypes with TRD on the whole genome when enough informative offspring are available for the existing alleles under analysis.

Convergence of the Models. The convergence was checked by visual inspection showing a fast convergence to the simulated TRD in the first iterations when relevant TRD is significantly observed. The effective chain size obtained for each sample was on average $10 \%$ of the total chain and decreased with the increase in the number of parameters (i.e., the number of heterozygous pairwise combinations, parameterization of parent-specific TRD) involved in the analysis. Moreover, even if a small effective chain was obtained, the estimated values were still accurate. It should be noted that the models were simple and the convergence to the estimated TRD value was fast, and therefore, high autocorrelation between iterations after convergence was observed. However, large number of iterations will be required when more parameters are involved in the model for more accurate estimations.

\section{Evaluation of TRD Models on Real Data}

SNP-by-SNP Analysis. Results across the whole genome displayed $865 \mathrm{SNP}$ with TRD that exceeded the $\mathrm{BF}$ threshold for strong evidence $(\mathrm{BF} \geq 10)$. The number of regions with parent-unspecific TRD was 637 SNP, whereas 315 and 368 SNP were detected as sireand dam-TRD, respectively. A total of 86 SNP were significantly detected as overall and sire- and damTRD. In this case, the magnitudes of the overall and the sire- and dam-specific TRD were similar and were mostly influenced by the number of informative offspring from the specific parent. The latter shows that the overall TRD also can be captured as parent-specific TRD when sufficient informative data are available for the specific parent. Notice that the model with parentunspecific TRD merges both sources of information (from sires and dams) to estimate one overall TRD. For regions with parent-specific TRD, 139 and 176 SNP with sire-TRD and dam-TRD were also detected as overall TRD, respectively, but with lower TRD values. If there are enough informative offspring for the parent with no observed TRD, this can confirm the parentorigin of TRD even if it is identified as overall TRD as well. This result highlights the importance of modeling the probability of inheritance considering the paternal 
Table 1. Average percentage of transmission ratio distortion (TRD) significantly detected by a Bayes factor $\geq 10$ and average mean absolute error (MAE), with the corresponding $95 \%$ confidence margin (biallelic loci; 3,000 replicates)

\begin{tabular}{|c|c|c|c|c|}
\hline \multirow{2}{*}{$\begin{array}{l}\text { No. of } \\
\text { informative } \\
\text { offspring }^{1}\end{array}$} & \multicolumn{2}{|c|}{ Overall TRD } & \multicolumn{2}{|c|}{ Sire/dam TRD } \\
\hline & $\begin{array}{l}\% \text { TRD } \\
\text { detected }\end{array}$ & MAE & $\begin{array}{l}\% \text { TRD } \\
\text { detected }\end{array}$ & MAE \\
\hline 10 & 30.93 & $0.060( \pm 0.0032)$ & 15.87 & $0.070( \pm 0.0048)$ \\
\hline 25 & 52.70 & $0.045( \pm 0.0018)$ & 44.70 & $0.055( \pm 0.0024)$ \\
\hline 50 & 63.93 & $0.034( \pm 0.0013)$ & 59.87 & $0.042( \pm 0.0016)$ \\
\hline 100 & 73.43 & $0.025( \pm 0.0009)$ & 68.43 & $0.032( \pm 0.0011)$ \\
\hline 200 & 81.13 & $0.018( \pm 0.0006)$ & 77.63 & $0.022( \pm 0.0008)$ \\
\hline 500 & 87.37 & $0.012( \pm 0.0004)$ & 85.80 & $0.015( \pm 0.0005)$ \\
\hline
\end{tabular}

${ }^{1}$ Informative offspring: offspring from one or both heterozygous parents for the overall TRD and from the specific heterozygous parent for the parent-specific TRD.

and maternal origin to capture parent-specific TRD. It is important to note that some biological mechanisms causing TRD can be limited to one of the parental sexes. Sex-dependent TRD has been previously reported in other studies (e.g., Alcaide et al., 2012; Liu et al., 2013; Didion et al., 2015; Casellas et al., 2017). In addition, 4 SNP were detected with sire- and dam-TRD in the opposite direction of preference of transmission. The latter could produce uncertainty in the direction of TRD for each parent given the interaction of both parameters in a large number of trios, but more likely it could suggest a possible interaction of alleles in the offspring genotypes (e.g., heterozygote advantage).

Regarding the statistical significance (i.e., BF), 416, 181, and 86 SNP were detected for overall TRD with a BF of $100,10^{5}$, and $10^{10}$, respectively. For sire-TRD, 185,87 , and 49 SNP were identified with a BF of 100 , $10^{5}$, and $10^{10}$, respectively, whereas 204,70 , and 32 SNP were detected for dam-TRD (BF $\geq 100, \geq 10^{5}$, and $\geq 10^{10}$, respectively). Moreover, several SNP showed extremely large $\mathrm{BF}\left[\log _{10}(\mathrm{BF})>100\right]$. These were highlighted by the magnitude of TRD and the number of informative offspring, supporting the relevance of these regions among others. From a biological perspective, the probability of null TRD for these regions can be considered 0.

Approximate Empirical Null Distribution of $T R D$. Despite the large number of regions detected with $\mathrm{BF} \geq 10$, most of them showed low $(<0.05)$ or extremely low magnitude of $|\mathrm{TRD}|$. It is important to remember that the magnitude of TRD determines how strongly one allele is over- or under-transmitted to the next generation. Whereas an $\alpha=0.5$ (or -0.5 ) means a full skewed transmission of one single allele, null TRD $(\alpha=0)$ keeps an equal probability of transmission from parents to offspring. It has been shown that some biological mechanisms, such as germline selection, meiotic drive, or gametic competition, differ from embryo lethality, because the disadvantaged genotype is still observable in the offspring generation (Huang et al., 2013). Thus, a low magnitude of TRD could be a consequence of some of these biological mechanisms that lead to observable disadvantaged genotypes or could also be the result of a sampling process of the offspring's genomes. For example, we observed that the same SNP that displayed significant TRD in a sample

Table 2. Average percentage of transmission ratio distortion (TRD) significantly detected by a Bayes factor $\geq 10$ and average mean absolute error (MAE) for different sample size of trios (n), with the corresponding 95\% confidence margin (multiallelic loci; sex ratio = 1:25; 3,000 replicates)

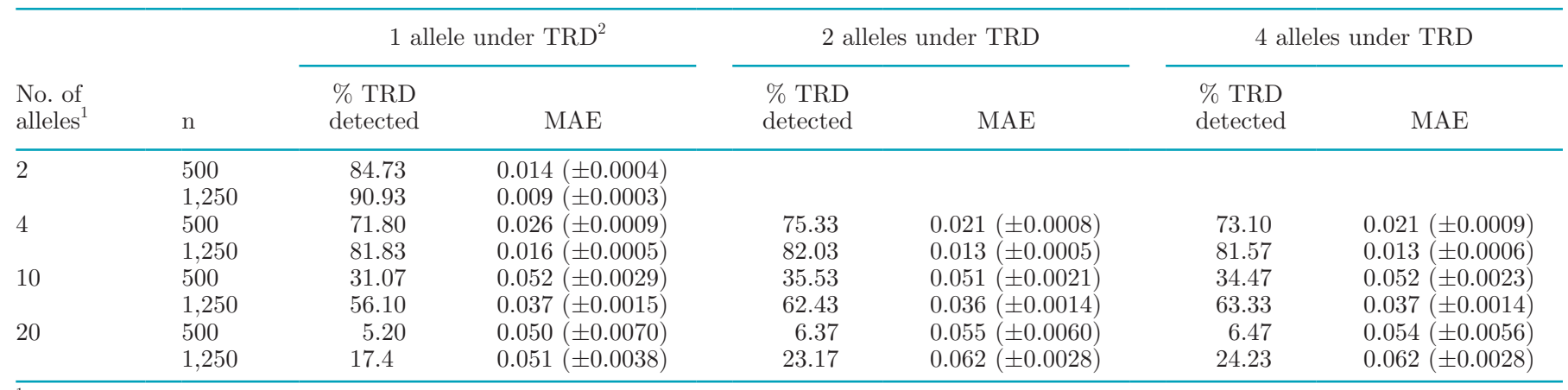

\footnotetext{
${ }^{1}$ No. of alleles: number of alleles at loci.
}

${ }^{2}$ Number of alleles under TRD at loci. 
of individuals showed no TRD when analyzed in the whole sample, evidencing the possibility of random TRD due to the sampling process in the offspring generation. It is important to acknowledge that even with a random sample of individuals, some SNP could end up with altered deviations in segregation just by chance. In fact, a parametric bootstrap approach, as described by Casellas et al. (2014), can partly correct for this effect by creating the expected distribution of the BF under no TRD for a specific marker. However, given that resampling is based on an individual SNP and does not consider the effect of the joint inheritance of large haplotypes or even gametes from chromosomes with few or no recombinations, it was not very powerful to discard random TRD.

Within this context, an approximate empirical null distribution of TRD to account for random TRD by resampling individuals was proposed. This distribution traced the generated TRD in a sample of individuals in relation to the whole available population sample. Given that the error in the TRD estimation decreased with the sample size, more random TRD was expected in small sample, making it important to construct different distributions for several sample sizes. By bounding a minimum threshold from these distributions for the estimated TRD, it would be possible to discard possible random TRD lacking other evidence supporting the observed TRD (Figure 3; Appendix Table A1). Taking into account this null distribution of TRD, the number of detected regions with overall TRD was reduced from 673 to $662,599,298$, and 71 with margins of error of $1,0.1,0.01$, and $0.001 \%$, respectively. For sire-TRD the number was reduced from 315 to 311 (1\%), 270 (0.1\%), $203(0.01 \%)$, and $64(0.001 \%)$ and from 368 to $359(1 \%), 308(0.1 \%), 223(0.01 \%)$, and $42(0.001 \%)$ for dam-TRD. In addition, a sizeable part of TRD regions that were discarded as random TRD (163 SNP for overall TRD) presented low $\mathrm{BF}<100$, basically due to the limited number of informative offspring, where the chance to generate random TRD is higher. It is important to note that the maximum $|\mathrm{TRD}|$ generated by chance with a sample size of $\sim 50,000$ informative offspring was 0.02 . A random $|\mathrm{TRD}|$ of up to 0.44 was observed when the number of informative offspring was $\leq 15$. On the other hand, TRD has been shown to be a confounding signal in linkage or association analysis (Huang et al., 2013). Therefore, this result could highlight the prevalence of the random TRD noticeably observed on small sample sizes that can be crucial in genetic studies.

Genotyping Error and Imputation. Genotyping errors are another source of bias associated with

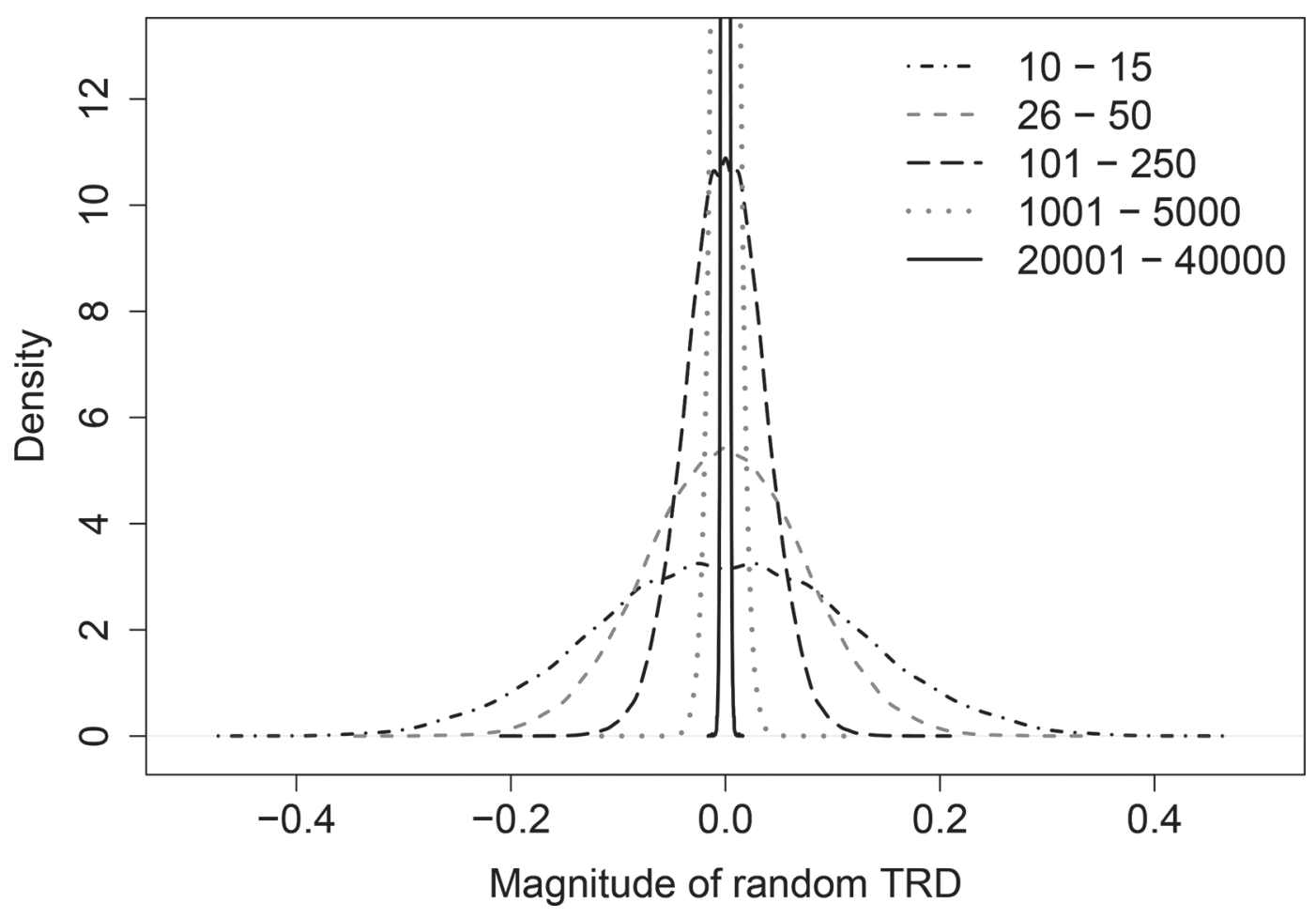

Figure 3. The approximate empirical null distribution of transmission ratio distortion (TRD) according to different ranges of the number of informative offspring (see Appendix for more details). Informative offspring: offspring from one or both heterozygous parents for the overall TRD and from the specific heterozygous parent for the parent-specific TRD. 
limitations on genotyping and sequencing technologies together with the reference genome itself, which can potentially produce important artifacts on TRD analyses. A false positive can be created easily if, for example, a homozygous parent was genotyped incorrectly as heterozygous, which would show a full deviation in the transmission of one allele, whereas the second allele does not really exist. This issue can have more of an effect for markers with few informative parents, and it is important to consider a minimal number of them to exclude false TRD. Particularly for sires, considering that one sire can have an important proportion of informative offspring. Thus, to verify if TRD is generated only from few heterozygous sires with a large number of offspring is important to avoid possible artifacts of genotyping errors. Indeed, it was reported by several authors that genotyping errors are an important confounder factor in this kind of analysis (e.g., Paterson et al., 2009; Meyer et al., 2012). In this study, genotyping errors leading to genotype incompatibilities between offspring and parents were accounted for. However, genotyping errors associated with heterozygous parents still could be an important source of bias.

Imputation could partially correct the problem of genotyping errors by integrating all the genomic and pedigree information together to accurately predict the missing genotypes and correcting for possible errors. Imputation affected TRD estimations in 2 ways. First, part of regions previously identified with TRD showed no TRD when imputed data were used $(451,167$, and 239 SNP for overall and sire- and dam-TRD, respectively). And also, most of these SNP had a small magnitude of TRD $(|\alpha|<0.05)$. This result could indicate possible TRD generated by genotyping error or random TRD, which resulted to be null when more informative and accurate data were provided by imputation. In addition, some of the regions displaying no TRD with imputed data could be due to inaccurate imputation. It should be noted that some SNP initially presented few genotyped trios (e.g., $32 \mathrm{SNP}$ with $<6,000$ trios), which to impute an additional $\sim 50,000$ trios can result in inaccurate imputation given the limited training data set. On the other side, by recovering missing genotyped data, providing more statistical power for TRD analyses, additional regions were detected with imputed data $(122,125$, and 31 for overall and sire- and dam-TRD, respectively). Furthermore, regions detected with both imputed and nonimputed data were 222, 148, and 129 for overall and sire- and dam-TRD, respectively. Based on these results, accurate imputation could minimize the effect of genotyping errors in TRD analyses.

TRD Effects. The SNP identified with moderateto-high $|\mathrm{TRD}|(\geq 0.15)$ showed extremely low frequency $(<0.008)$, evidencing the effect of TRD selection. Note that TRD is a "selfish" genetic element that provides a transmission advantage for one of the alleles, which can result in the spread of the allele through the population and eventually its fixation (Hurst and Werren, 2001). Although a low frequency was observed for these SNP, the number of individuals carrying the minor allele was still significant (e.g., 55 heterozygous sires, 365 heterozygous dams, and 2,255 informative offspring for the SNP with a frequency of 0.008). Nevertheless, from a population genetics perspective, the maintenance of a disadvantaged allele in the population can be caused by some countervailing force, such as recombination (Haig and Grafen, 1991), mutation, genetic drift, and selection (Polański et al., 1998), and an immunogenetic advantage for survival regardless of the posterior impaired fertility (Westendorp et al., 2001). Note that the alleles with advantage transmission are not always beneficial, but can show deleterious fitness effects resulting in the maintenance of the polymorphisms (Carvalho and Vaz, 1999), such as in the mouse t-haplotype system and the segregation distorter system in Drosophila.

On the other hand, parent-specific TRD model identified genomic regions where moderate-to-high $|T R D|$ were observed for sire-TRD in more polymorphic regions (frequency $\sim 0.03$ ). The maintenance of this polymorphism in these regions could suggest some countervailing forces between paternal and maternal transmissions leading to fixation via one single parent and its maintenance in the opposite sex. In addition, these rare variants captured by TRD are displayed on interesting regions of the genome that can be associated with complex or recessive disorders, which can be difficult to capture by genome-wide association studies.

Another source of bias that may affect TRD analyses, as previously introduced, is the nonrandom selection within families [i.e., pre-selection of offspring to be genotyped (Id-Lahoucine and Casellas, 2017)]. The genetic architecture of the trait under single- or multiple-trait selection may also have different effects in the generation of artifacts of TRD across the genome. For instance, the effect could be minimal for highly polygenic traits, whereas it may increase in the presence of major genes. Within this context, the criteria for choosing offspring within families for genotyping should be acknowledged and taken into consideration to properly address the TRD analyses and the corresponding results and interpretations. To protect against the potential selection biases, strategies such as considering a maximum offspring age at genotyping could be a feasible solution. Notice that animals genotyped at an early age are unlikely to be selected for a specific phenotype in comparison to animals genotyped at an older age. 
Haplotype Analysis. The implementation of haplotype in TRD analyses could allow discovering segments of the genome subject to the TRD phenomenon. Using windows of SNP to scan the genome, we were able to identify regions that included the SNP already identified from the SNP-based method as new genomic regions. Figure 4 showed the magnitude of TRD and the $\mathrm{BF}$ for the regions identified for both SNP- and haplotype-based method for 3 chromosomes (covered by $7,421 \mathrm{SNP}$ ). Within this context, haplotype analysis was advantageous by targeting more precisely the genomic areas underlying the TRD. It was observed that higher estimated TRD was found in regions that included the SNP already identified with small TRD in SNP-by-SNP analysis, but showing more specifically the haplotype allele generating TRD in the cor- responding region. These haplotype alleles were more likely to harbor the causal mutations in comparison to individual SNP, which may be in low linkage disequilibrium with the causal variant. For example, the first peak highlighted of chromosome 2, in Figure 4, corresponds to a SNP and 10-SNP haplotype including the same SNP showing magnitudes of TRD of -0.022 and -0.411 , respectively. Moreover, the likelihood that a genotyping error in a SNP that displayed a TRD in some heterozygous parents will be linked by chance to one single haplotype allele is low. Thus, identification of one haplotype allele fully explaining the observed TRD in the included SNP could support the TRD over possible genotyping errors.

On the other hand, by increasing the length of the haplotype, more significant regions were identified (BF
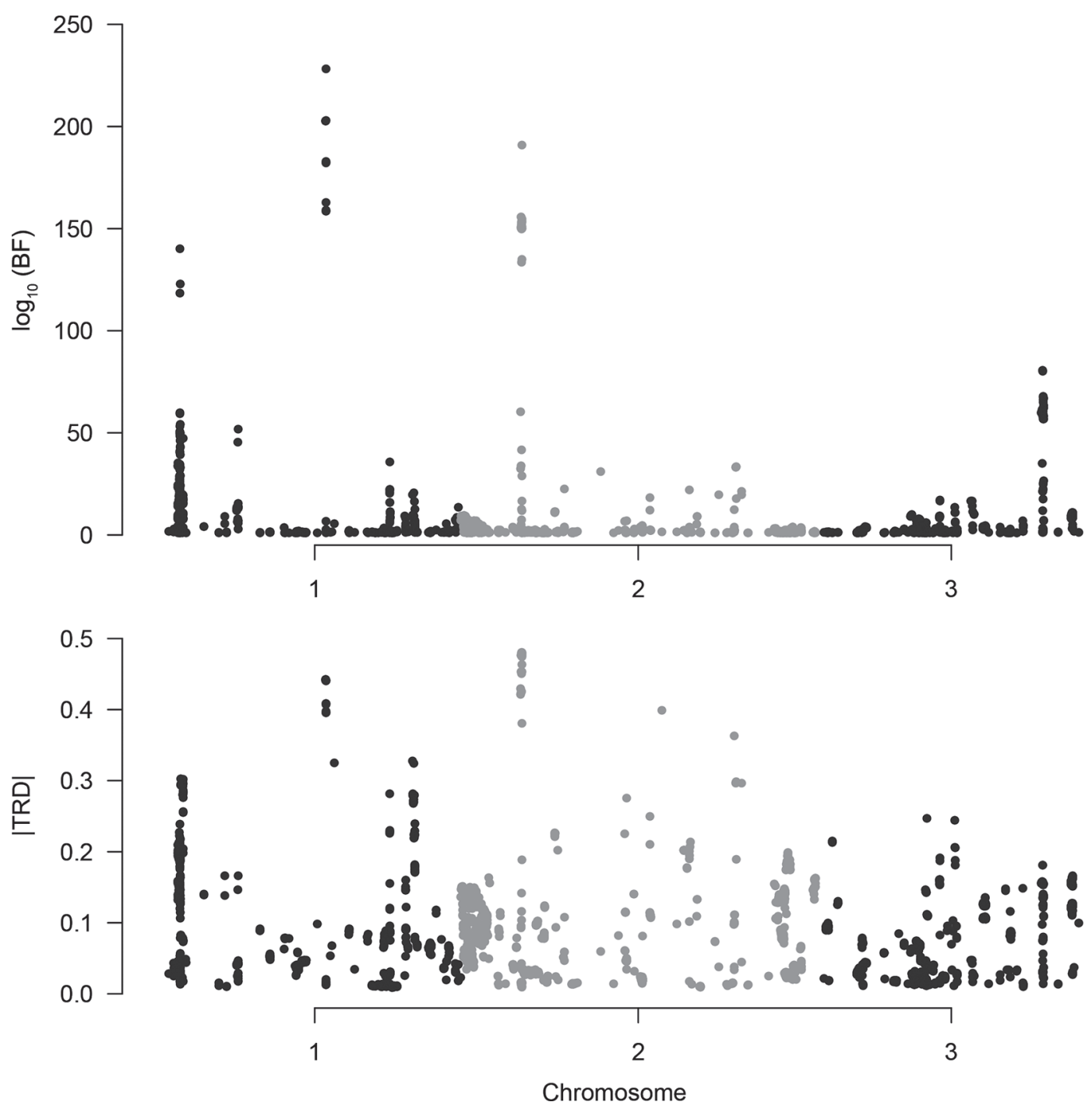

Figure 4. Bayes factor (BF) and magnitude of transmission ratio distortion (TRD) of SNP and haplotypes of 2, 4, 7, 10, and 20 SNP significantly detected with $\mathrm{BF} \geq 10$ across 3 chromosomes of Holstein genotypes. 
$\geq 10$ ). In Figure 4 , regions identified with $\mathrm{BF} \geq 10$ were $53,150,316,623,819$, and 1,176 for SNP and 2-, 4-, 7-, 10-, and 20-SNP haplotype-based methods, respectively. Considering a decisive evidence threshold (BF $\geq 100$ ), reduced to $28,69,160,295,378$, and 649 regions for SNP and 2-, 4-, 7-, 10-, and 20-SNP haplotypebased methods, respectively. By discarding random TRD at $<0.001 \%$, the number of region reduced to 11 , 19, 43, 64, 88, and 153 for SNP and 2-, 4-, 7-, 10-, and 20-SNP haplotype-based methods, respectively. Within this context, the haplotype-based method showed more ability to capture TRD compared with individual SNP. In fact, SNP arrays are usually designed with a bias toward high MAF, which may display low level of linkage disequilibrium with low frequency causal variants. The haplotype-based methods exploit the already available SNP arrays, providing an additional range of allele frequencies across the whole genome, resulting in a more powerful method to unravel TRD. In addition, more polymorphic regions with moderate-to-high $|\mathrm{TRD}|$ tended to show in haplotypes rather than individual SNP.

Integration of the results from SNP-by-SNP and different haplotype lengths analyses will be required to detect the core regions of TRD. Within this context, considering the chromosomes of Figure 4, 44 regions with significance TRD across these 3 chromosomes can be differentiated. The SNP or haplotype allele with higher $\mathrm{BF}$ will correspond to the core allele of the region. Additionally, restricting a minimal number of informative parents and a multiple test correction by selecting the top regions according the BF could provide a more promising region in terms of magnitude of TRD and number of informative offspring.

Regarding the performance of the 2 procedures of haplotype analyses, both methods showed comparable results with some singular differences. First, regions identified only by heterozygous pairwise combinations procedure could be explained by a specific interaction of alleles in parental generation, TRD observed in some families, or just a random TRD given the low statistical power available for each heterozygous pairwise combination. Second, when using the biallelic haplotype procedure, merging all heterozygous pairwise combinations together in one single analysis for the common allele increased the statistical power, resulting in better ability to detect regions previously undetectable using the heterozygous pairwise combination method. When the results of both methods were similar, several heterozygous pairwise combinations with a specific common allele showed a similar magnitude of TRD (influenced by the number of informative individuals) to that of the common allele when analyzed independently (e.g., 3 combinations displaying magnitude of TRD of -0.06 ,
-0.10 , and -0.08 , whereas it was -0.08 for the common haplotype allele in the biallelic haplotype procedure).

Finally, it is important to note that the computing time increases exponentially with the length of haplotypes (number of possible alleles). Therefore, it is necessary to consider the computational time to determine the length of windows to scan the whole genome. Short windows can provide signals of TRD, which could be subsequently target with large haplotypes to detect more precisely the haplotype carrying the allele associated with TRD. However, the second procedure of haplotype analysis based on remodeling the multiallelic loci to biallelic loci did not present this limitation and allowed estimation of TRD parameters in a more rational computational time. In addition, the developed program allows specifying a minimal number of informative offspring, sires, or dams to perform the analyses, being a practical strategy to reduce the computational time.

Insights into the Biological Implications of $T R D$. Functional analyses of the identified regions with TRD suggested positional candidate genes related to crucial biological processes associated with embryonic development and survival, such as the large tumor suppressor kinase 2 (LATS2) gene (McPherson et al., 2004; Powzaniuk et al., 2004), BUB1 mitotic checkpoint serine/threonine kinase (BUB1) gene (Leland et al., 2009 ), and nibrin $(N B N)$ gene (Warcoin et al., 2009), as well as genes related to sperm deformation [e.g., spermatid maturation 1 (SPEM1; Zheng et al., 2007)]. To substantiate the TRD approach, previously known regions with lethal alleles were examined. Among them, patterns of TRD were identified on Holstein haplotype 2 (VanRaden et al., 2011; McClure et al., 2014), Holstein haplotype 4 (Fritz et al., 2013), Holstein haplotype 5 (Schütz et al., 2016), and Holstein haplotype D (Shanks et al., 1984). Also, signals of TRD were identified in the Holstein data analyzed that had previously been discovered in other breeds, such as Jersey haplotype 1 (Eldridge et al., 1951; Duchesne et al., 2006) and haplotype BHM in Brown Swiss (el-Hamidi et al., 1989; Krebs et al., 2007).

In addition, several QTL from Cattle QTL database (CattleQTLdb, www.animalgenome.org/cgi-bin/ QTLdb/BT/index) were detected overlapping with TRD regions. Among them, 1,091 QTL associated with reproductive traits were located in 357 genomic regions of $100 \mathrm{~kb}$ (50 kb downstream and upstream) displaying TRD. Among the reproductive traits, conception rate (Parker Gaddis et al., 2016), calving ease (Cole et al., 2011), and stillbirth and birth index traits (Höglund et al., 2012) had QTL located in the 100-kb genomic regions displaying TRD with the highest BF. These results support the relevance of TRD analyses. Future 
publications more deeply investigating the genomic regions with TRD with respect to their biological and functional implications are anticipated.

\section{Applications}

The Bayesian analyses of TRD showed high accuracy and statistical power to detect regions (SNP and haplotypes) with overall or sire- and dam-specific TRD at a genome-wide scale. TRDscan v.1.0 provides a powerful statistical tool for scanning for TRD regions across the whole genome, allowing for their characterization and discovery of candidate genomic regions for further investigation. This program includes SNP-by-SNP analysis and a haplotype-based method with 2 procedures of analyses (i.e., heterozygous pairwise combinations and biallelic haplotype). The program also provides additional information for TRD analyses as the number of informative parents and offspring, frequencies, probabilities of random TRD, and so on. The developed program and the user's guide will be available at http: //www.casellas.info/files/TRDscan.zip.

\section{ACKNOWLEDGMENTS}

This research was funded by Beef Farmers Ontario (BFO-16-04; Guelph, Canada), Mitacs accelerate (Toronto, Canada), The Semex Alliance (Guelph, Canada), and through the Canadian Beef Science Cluster by the Beef Cattle Research Council (Calgary, Canada) and Agriculture and Agri-Food Canada (Guelph, Canada). The authors acknowledge the data provided by the Canadian Dairy Network (Guelph, ON).

\section{REFERENCES}

Agulnik, S. I., A. I. Agulnik, and A. O. Ruvinsky. 1990. Meiotic drive in female mice heterozygous for the HSR insert on chromosome 1. Genet. Res. 55:97-100.

Alcaide, M., A. Rodríguez, J. J. Negro, and D. Serrano. 2012. Male transmission ratio distortion supports MHC-linked cryptic female choice in the lesser kestrel (Aves: Falconidae). Behav. Ecol. Sociobiol. 66:1467-1473. https://doi.org/10.1007/s00265-012-1401-9.

Axelsson, E., A. Albrechtsen, A. P. van, L Li, H. J. Megens, A. L. Vereijken, R. P. Crooijmans, M. A. Groenen, H. Ellegren, E. Willerslev, and R. Nielsen. 2010. Segregation distortion in chicken and the evolutionary consequences of female meiotic drive in birds. Heredity 105:290-298. https://doi.org/10.1038/hdy.2009.193.

Boyd, G. W. 1993. Bull Power: Productivity and Profitability. Range Beef Cow Symposium. Paper 221. Cheyenne, Wyoming. Accessed Jan. 21, 2019. http://digitalcommons.unl.edu/rangebeefcowsymp/ 221.

Carvalho, A. B., and S. C. Vaz. 1999. Are Drosophila SR drive chromosomes always balanced? Heredity 83:221-228. https://doi.org/ 10.1038 /sj.hdy. 6886100 .

Casellas, J. 2007. Survival quantitative trait locus fine mapping by measuring and testing for Hardy-Weinberg and linkage disequilibrium. Genetics 176:721-724. https://doi.org/10.1534/genetics.106 .067264 .
Casellas, J., J. J. Cañas-Álvarez, A. González-Rodríguez, A. Puig-Oliveras, M. Fina, J. Piedrafita, A. Molina, C. Díaz, J. A. Baró, and L. Varona. 2017. Bayesian analysis of parent-specific transmission ratio distortion in seven Spanish beef cattle breeds. Anim. Genet. 48:93-96. https://doi.org/10.1111/age.12509.

Casellas, J., R. J. Gularte, C. R. Farber, L. Varona, M. Mehrabian, E. E. Schadt, A. J. Lusis, A. D. Attie, B. S. Yandell, and J. F. Medrano. 2012. Genome scans for transmission ratio distortion regions in mice. Genetics 191:247-259. https://doi.org/10.1534/ genetics.111.135988.

Casellas, J., A. Manunza, A. Mercader, R. Quintanilla, and M. Amills. 2014. A flexible Bayesian model for testing for transmission ratio distortion. Genetics 198:1357-1367. https://doi.org/10.1534/ genetics.114.169607.

Cole, J. B., G. R. Wiggans, L. Ma, T. S. Sonstegard, T. J. Lawlor Jr., B. A. Crooker, C. P. Van Tassell, J. Yang, S. Wang, L. K. Matukumalli, and Y. Da. 2011. Genome-wide association analysis of thirty one production, health, reproduction and body conformation traits in contemporary U.S. Holstein cows. BMC Genomics 12:408.

Dean, N. L., J. C. Loredo-Osti, T. M. Fujiwara, K. Morgan, S. L. Tan, A. K. Naumova, and A. Ao. 2006. Transmission ratio distortion in the myotonic dystrophy locus in human preimplantation embryos. Eur. J. Hum. Genet. 14:299-306. https://doi.org/10.1038/sj.ejhg .5201559 .

Didion, J. P., A. P. Morgan, A. M. F. Clayshulte, R. C. Mcmullan, L. Yadgary, P. M. Petkov, T. A. Bell, D. M. Gatti, J. J. Crowley, K. Hua, D. L. Aylor, L. Bai, M. Calaway, E. J. Chesler, J. E. French, T. R. Geiger, T. J. Gooch, T. Garland, A. H. Harrill, K. Hunter, L. McMillan, M. Holt, D. R. Miller, D. A. O'Brien, K. Paigen, W. Pan, L. B. Rowe, G. D. Shaw, P. Simecek, P. F. Sullivan, K. L. Svenson, G. M. Weinstock, D. W. Threadgill, D. Pomp, G. A. Churchill, and F. Pardo-Manuel de Villena. 2015. A multi-megabase copy number gain causes maternal transmission ratio distortion on mouse chromosome 2. PLoS Genet. 11:e1004850.

Duchesne, A., M. Gautier, S. Chadi, C. Grohs, S. Floriot, Y. Gallard, G. Caste, A. Ducos, and A. Eggen. 2006. Identification of a doublet missense substitution in the bovine LRP4 gene as a candidate causal mutation for syndactyly in Holstein cattle. Genomics $88: 610-621$

Dyer, K. A., B. Charlesworth, and J. Jaenike. 2007. Chromosome-wide linkage disequilibrium as a consequence of meiotic drive. Proc. Natl. Acad. Sci. USA 104:1587-1592.

el-Hamidi, M., H. W. Leipold, J. G. E. Vestweber, and G. Saperstein. 1989. Spinal muscular atrophy in Brown Swiss calves. J. Vet. Med. A Physiol. Pathol. Clin. Med. 36:731-738.

Eldridge, F. E., W. H. Smith, and W. M. McLeod. 1951. Syndactylism in Holstein-Friesian cattle: Its inheritance, description and occurrence. J. Hered. 42:241-250.

Evans, K., A. Fryer, C. Inglehearn, J. Duvall-Young, J. L. Whittaker, C. Y. Gregory, R. Butler, N. Ebenezer, D. M. Hunt, and S. Bhattacharya. 1994. Genetic linkage of cone-rod retinal dystrophy to chromosome 19q and evidence for segregation distortion. Nat. Genet. 6:210-213. https://doi.org/10.1038/ng0294-210.

Eversley, C. D., T. Clark, Y. Xie, J. Steigerwalt, T. A. Bell, F. P. M. de Villena, and D. W. Threadgill. 2010. Genetic mapping and developmental timing of transmission ratio distortion in a mouse interspecific backcross. BMC Genet. 11:98. https://doi.org/10.1186/ 1471-2156-11-98

Fritz, S., A. Capitan, A. Djari, S. C. Rodriguez, A. Barbat, A. Baur, C. Grohs, B. Weiss, M. Boussaha, D. Esquerré, C. Klopp, D. Rocha, and D. Boichard. 2013. Detection of haplotypes associated with prenatal death in dairy cattle and identification of deleterious mutations in GART, SHBG and SLC37A2. PLoS One 8:e65550.

García-Cortés, L. A., C. Cabrillo, C. Moreno, and L. Varona. 2001. Hypothesis testing for the genetic background of quantitative traits. Genet. Sel. Evol. 33:3-16.

Gelfand, A., and A. Smith. 1990. Sampling based approaches to calculating marginal densities. J. Am. Stat. Assoc. 85:398-409.

Geyer, C. J. 1992. Practical Markov chain Monte Carlo. Stat. Sci $7: 473-511$ 
Haig, D., and A. Grafen. 1991. Genetic scrambling as a defence against meiotic drive. J. Theor. Biol. 153:531-558.

Hall, M. C., and J. H. Willis. 2005. Transmission ratio distortion in intraspecific hybrids of Mimulus guttatus: Implications for genomic divergence. Genetics 170:375-386. https://doi.org/10.1534/ genetics.104.038653.

Hastings, I. M. 1991. Germline selection: Population genetic aspects of the sexual/asexual life cycle. Genetics 129:1167-1176.

Hastings, W. K. 1970. Monte Carlo sampling methods using Markov chains and their applications. Biometrika 57:97-109. https://doi .org $/ 10.2307 / 2334940$.

Hoff, J. L., J. E. Decker, R. D. Schnabel, and J. F. Taylor. 2017. Candidate lethal haplotypes and causal mutations in Angus cattle. BMC Genomics 18:799. https://doi.org/10.1186/s12864-017-4196 -2 .

Höglund, J. K., B. Guldbrandtsen, M. S. Lund, and G. Sahana. 2012. Analyzes of genome-wide association follow-up study for calving traits in dairy cattle. BMC Genet. 13:71.

Huang, L. O., A. Labbe, and C. Infante-Rivard. 2013. Transmission ratio distortion: A review of concept and implications for genetic association studies. Hum. Genet. 132:245-263. https://doi.org/10 .1007/s00439-012-1257-0.

Hurst, G. D. D., and J. H. Werren. 2001. The role of selfish genetic elements in eukaryotic evolution. Nat. Rev. Genet. 2:597-606. https: //doi.org/10.1038/35084545.

Id-Lahoucine, S., and J. Casellas. 2017. Impact of incomplete pedigree data and independent culling level pre-selection on the genetic evaluation of livestock: A simulation study on lamb growth. Livest. Sci. 198:76-81. https://doi.org/10.1016/j.livsci.2017.02.011.

Jeffreys, H. 1984. Theory of Probability. Clarendon Press, Oxford, UK.

Kass, R. E., and A. E. Raftery. 1995. Bayes factors. J. Am. Stat. Assoc. 90:773-795.

Krebs, S., I. Medugorac, S. Röther, K. Strässer, and M. Förster. 2007. A missense mutation in the 3-ketodihydrosphingosine reductase FVT1 as candidate causal mutation for bovine spinal muscular atrophy. Proc. Natl. Acad. Sci. USA 104:6746-6751.

Leland, S., P. Nagarajan, A. Polyzos, S. Thomas, G. Samaan, R. Donnell, F. Marchetti, and S. Venkatachalam. 2009. Heterozygosity for a Bub1 mutation causes female-specific germ cell aneuploidy in mice. Proc. Natl. Acad. Sci. USA 106:12776-12781. https://doi .org/10.1073/pnas.0903075106.

Liu, Y., L. Zhang, S. Xu, L. Hu, L. D. Hurst, and X. Kong. 2013. Identification of two maternal transmission ratio distortion loci in pedigrees of the Framingham heart study. Sci. Rep. 3:2147. https: //doi.org/10.1038/srep02147.

Lyon, M. F. 1991. The genetic basis of transmission-ratio distortion and male sterility due to the $t$ complex. Am. Nat. 137:349-358.

McClure, M. C., D. Bickhart, D. Null, P. VanRaden, L. Xu, G. Wiggans, G. Liu, S. Schroeder, J. Glasscock, J. Armstrong, J. B. Cole, C. P. Van Tassell, and T. S. Sonstegard. 2014. Bovine exome sequence analysis and targeted SNP genotyping of recessive fertility defects $\mathrm{BH} 1, \mathrm{HH} 2$, and $\mathrm{HH} 3$ reveal causative mutation in SMC2 for HH3. PLoS One 9:e92769.

McPherson, J. P., L. Tamblyn, A. Elia, E. Migon, A. Shehabeldin, E. Matysiak-Zablocki, B. Lemmers, L. Salmena, A. Hakem, J. Fish, F. Kassam, J. Squire, B. G. Bruneau, M. P. Hande, and R. Hakem. 2004. Lats2/Kpm is required for embryonic development, proliferation control and genomic integrity. EMBO J. 23:3677-3688.

Meyer, W. K., B. Arbeithuber, C. Ober, T. Ebner, I. Tiemann-Boege, R. R. Hudson, and M. Przeworski. 2012. Evaluating the evidence for transmission distortion in human pedigrees. Genetics 191:215232. https://doi.org/10.1534/genetics.112.139576.

Moore, C. S. 2006. Postnatal lethality and cardiac anomalies in the Ts65Dn Down Syndrome mouse model. Mamm. Genome 17:10051012. https://doi.org/10.1007/s00335-006-0032-8.

Nadeau, J. H. 2017. Do gametes woo? Evidence for their nonrandom union at fertilization. Genetics 207:369-387.

Naumova, A. K., C. M. Greenwood, and K. Morgan. 2001. Imprinting and deviation from Mendelian transmission ratios. Genome 44:311-320.
Pardo-Manuel de Villena, F., E. de la Casa-Esperon, T. L. Briscoe, and C. Sapienza. 2000. A genetic test to determine the origin of maternal transmission ratio distortion. Meiotic drive at the mouse Om locus. Genetics 154:333-342.

Parker Gaddis, K. L., D. J. Null, and J. B. Cole. 2016. Explorations in genome-wide association studies and network analyses with dairy cattle fertility traits. J. Dairy Sci. 99:6420-6435.

Paterson, A. D., D. Waggott, A. Schillert, C. Infante-Rivard, S. B. Bull, Y. J. Yoo, and D. Pinnaduwage. 2009. Transmission-ratio distortion in the Framingham Heart Study. BMC Proc. 3(Suppl. 7):S51. https://doi.org/10.1186/1753-6561-3-S7-S51.

Polański, A., R. Chakraborty, M. Kimmel, and R. Deka. 1998. Dynamic balance of segregation distortion and selection maintains normal allele sizes at the myotonic dystrophy locus. Math. Biosci. 147:93-112.

Powzaniuk, M., S. McElwee-Witmer, R. L. Vogel, T. Hayami, S. J. Rutledge, F. Chen, S. Harada, A. Schmidt, G. A. Rodan, L. P. Freedman, and C. Bai. 2004. The LATS2/KPM tumor suppressor is a negative regulator of the androgen receptor. Mol. Endocrinol. 18:2011-2023.

Sargolzaei, M., J. P. Chesnais, and F. S. Schenkel. 2014. A new approach for efficient genotype imputation using information from relatives. BMC Genomics 15:478. https://doi.org/10.1186/1471 -2164-15-478.

Schütz, E., C. Wehrhahn, M. Wanjek, R. Bortfeld, W. E. Wemheuer, J. Beck, and B. Brenig. 2016. The Holstein Friesian lethal haplotype 5 (HH5) results from a complete deletion of TFB1M and cholesterol deficiency (CDH) from an ERV-(LTR) insertion into the coding region of APOB. PLoS One 11:e0154602.

Shanks, R. D., D. B. Dombrowski, G. W. Harpestad, and J. L. Robinson. 1984. Inheritance of UMP synthase in dairy cattle. J. Hered. 75:337-340.

Silver, L. M. 1989. Gene dosage effects on transmission ratio distortion and fertility in mice that carry $t$ haplotypes. Genet. Res. $54: 221-225$.

Silver, L. M. 1993. The peculiar journey of a selfish chromosome: Mouse $t$ haplotypes and meiotic drive. Trends Genet. 9:250-254. https://doi.org/10.1016/0168-9525(93)90090-5.

Sun, C., P. M. VanRaden, J. R. O'Connell, K. A. Weigel, and D. Gianola. 2013. Mating programs including genomic relationships and dominance effects. J. Dairy Sci. 96:8014-8023. https://doi .org/10.3168/jds.2013-6969.

VanRaden, P. M., K. M. Olson, D. J. Null, and J. L. Hutchison. 2011. Harmful recessive effects on fertility detected by absence of homozygous haplotypes. J. Dairy Sci. 94:6153-6161.

Varona, L., L. A. García-Cortés, and M. Pérez-Enciso. 2001. Bayes factors for detection of quantitative trait loci. Genet. Sel. Evol. 33:133-152.

Wakasugi, N. 1974. A genetically determined incompatibility system between spermatozoa and eggs leading to embryonic death in mice. J. Reprod. Fertil. 41:85-96. https://doi.org/10.1530/jrf.0.0410085.

Warcoin, M., J. Lespinasse, G. Despouy, C. Dubois d'Enghien, A Laugé, M. F. Portnoï, S. Christin-Maitre, D. Stoppa-Lyonnet, and M. H. Stern. 2009. Fertility defects revealing germline biallelic nonsense NBN mutations. Hum. Mutat. 30:424-430. https://doi .org/10.1002/humu.20904.

Westendorp, R. G., F. M. van Dunne, T. Kirkwood, F. M. Helmerhorst, and T. Huizinga. 2001. Optimizing human fertility and survival. Nat. Med. 7:873

Yang, L., M. F. Andrade, S. Labialle, S. Moussette, G. Geneau, D. Sinnett, A. Belisle, C. M. Greenwood, and A. K. Naumova. 2008. Parental effect of DNA (cytosine-5) methyltransferase 1 on grandparentalorigin-dependent transmission ratio distortion in mouse crosses and human families. Genetics 178:35-45.

Zheng, H., C. J. Stratton, K. Morozumi, J. Jin, R. Yanagimachi, and W. Yan. 2007. Lack of Spem1 causes aberrant cytoplasm removal, sperm deformation, and male infertility. Proc. Natl. Acad. Sci. USA 104:6852-6857.

Zöllner, S., X. Wen, N. A. Hanchard, M. A. Herbert, C. Ober, and J. K. Pritchard. 2004. Evidence for extensive transmission distortion in the human genome. Am. J. Hum. Genet. 74:62-72. 


\section{APPENDIX}

For pedigrees with incomplete trios, Casellas et al. (2014) included an additional parameter (allelic frequency, $\pi)$ in the TRD model to allow estimating an overall TRD. Within this context, by assuming a parent-unspecific TRD and that all parents came from the same population, the model parameterized the probability of inheritance (P) from known parents as the model outlined above [i.e., $\mathrm{P}(\mathrm{A})=1-\mathrm{P}(\mathrm{B})=0.5+\alpha$ and $\mathrm{P}(\mathrm{B})=1-\mathrm{P}(\mathrm{A})=$ $0.5-\alpha]$ and appropriately modeling the probability of inheritance from ungenotyped parents as

$$
\mathrm{P}(\mathrm{A})=\pi \text { and } \mathrm{P}(\mathrm{B})=1-\pi,
$$

where $\pi$ is the allelic frequency.

In this case, the conditional posterior distribution of $\pi$ and $\alpha$ in Bayesian implementation becomes

$$
\mathrm{p}(\pi, \alpha \mid \mathbf{y}) \propto \mathrm{p}(\mathbf{y} \mid \pi, \alpha) \mathrm{p}(\pi) \mathrm{p}(\alpha)
$$

where $\pi$ is the allelic frequency, $\alpha$ is the overall TRD, and $\mathbf{y}$ is the vector of offspring's genotypes. Flat prior was also assumed for $\pi$ within a parametric space ranging from 0 to 1 .

To extend this model for parent-specific TRD model for biallelic loci and both models for multiallelic loci, prior knowledge on estimates of allele frequencies must be included to overcome the limited degrees of freedom available. Within the context, both parent-specific TRD and also the overall TRD could be estimated assuming the same parameterization for known parents but including known estimates of the frequencies $\left(\pi^{*}\right)$ for ungenotyped parents [i.e., $\mathrm{P}(\mathrm{A})=1-\mathrm{P}(\mathrm{B})=\pi^{*}$ ]. In Bayesian implementation, both models generalize to

$$
\mathrm{p}\left(\alpha \mid \mathbf{y}, \pi^{*}\right) \propto \mathrm{p}\left(\mathbf{y}, \pi^{*} \mid \alpha\right) \mathrm{p}(\alpha) \text { and } \mathrm{p}\left(\alpha_{\mathrm{s}}, \alpha_{\mathrm{d}} \mid \mathbf{y}, \pi^{*}\right) \propto \mathrm{p}\left(\mathbf{y}, \pi^{*} \mid \alpha_{\mathrm{s}}, \alpha_{\mathrm{d}}\right) \mathrm{p}\left(\alpha_{\mathrm{s}}\right) \mathrm{p}\left(\alpha_{\mathrm{d}}\right),
$$

where $\pi^{*}$ is the known allelic frequency, $\mathbf{y}$ is the vector of offspring's genotypes, and $\alpha, \alpha_{\mathrm{s}}$, and $\alpha_{\mathrm{d}}$ are the overall and sire- and dam-TRD parameters, respectively.

For multiallelic loci, the same generalization can be used. The conditional posterior distribution of the TRD parameters can be defined as

$$
\begin{aligned}
& \mathrm{p}\left(\alpha_{12}, \alpha_{13}, \ldots \alpha_{(\mathrm{n}-1) \mathrm{n}} \mid \mathbf{y}, \pi_{1}{ }^{*}, \pi_{2}{ }^{*}, \ldots \pi_{\mathrm{n}}{ }^{*}\right) \propto \mathrm{p}\left(\mathbf{y}, \pi_{1}{ }^{*}, \pi_{2}{ }^{*}, \ldots \pi_{\mathrm{n}}{ }^{*} \mid \alpha_{12}, \alpha_{13}, \ldots \alpha_{(\mathrm{n}-1) \mathrm{n}}\right) \mathrm{p}\left(\alpha_{12}\right) \mathrm{p}\left(\alpha_{13}\right) \ldots \mathrm{p}\left(\alpha_{(\mathrm{n}-1) \mathrm{n}}\right), \text { and } \\
& \mathrm{p}\left(\alpha_{\mathrm{s} 12}, \alpha_{\mathrm{d} 12}, \alpha_{\mathrm{s} 13}, \alpha_{\mathrm{d} 13}, \ldots \alpha_{\mathrm{s}(\mathrm{n}-1) \mathrm{n}}, \mathrm{\alpha}_{\mathrm{d}(\mathrm{n}-1) \mathrm{n}} \mid \mathbf{y}, \pi_{1}{ }^{*}, \pi_{2}{ }^{*}, \ldots \pi_{\mathrm{n}}{ }^{*}\right) \propto \mathrm{p}\left(\mathbf{y}, \pi_{1}{ }^{*}, \pi_{2}{ }^{*}, \ldots \pi_{\mathrm{n}}{ }^{*} \mid \alpha_{\mathrm{s} 12}, \alpha_{\mathrm{d} 12}, \alpha_{\mathrm{s} 13}, \alpha_{\mathrm{d} 13}, \ldots\right. \\
& \left.\alpha_{\mathrm{s}(\mathrm{n}-1) \mathrm{n}}, \alpha_{\mathrm{d}(\mathrm{n}-1) \mathrm{n}}\right) \mathrm{p}\left(\alpha_{\mathrm{s} 12}\right) \mathrm{p}\left(\alpha_{\mathrm{d} 12}\right) \mathrm{p}\left(\alpha_{\mathrm{s} 13}\right) \mathrm{p}\left(\alpha_{\mathrm{d} 13}\right) \ldots \mathrm{p}\left(\alpha_{\mathrm{s}(\mathrm{n}-1) \mathrm{n}}\right) \mathrm{p}\left(\alpha_{\mathrm{d}(\mathrm{n}-1) \mathrm{n}}\right),
\end{aligned}
$$

\begin{tabular}{|c|c|c|c|c|c|}
\hline \multirow{2}{*}{$\begin{array}{l}\text { No. of informative } \\
\text { offspring }^{1}\end{array}$} & \multicolumn{5}{|c|}{ Threshold on the null distribution of $|\mathrm{TRD}|^{2}$} \\
\hline & $5.00 \%$ & $1.00 \%$ & $0.10 \%$ & $0.01 \%$ & $0.001 \%$ \\
\hline $10-15$ & 0.25984 & 0.31972 & 0.37338 & 0.42537 & 0.44280 \\
\hline $16-25$ & 0.21534 & 0.27356 & 0.33114 & 0.38443 & 0.40823 \\
\hline $26-50$ & 0.16643 & 0.20618 & 0.26385 & 0.28420 & 0.32690 \\
\hline $51-100$ & 0.12199 & 0.15401 & 0.19566 & 0.22527 & 0.27830 \\
\hline $101-250$ & 0.08263 & 0.10646 & 0.13517 & 0.16558 & 0.20039 \\
\hline $251-1.000$ & 0.04907 & 0.06379 & 0.08580 & 0.11571 & 0.16335 \\
\hline $1,001-5,000$ & 0.02371 & 0.03162 & 0.04219 & 0.05362 & 0.11359 \\
\hline $5,001-20,000$ & 0.01022 & 0.01345 & 0.01776 & 0.02282 & 0.03390 \\
\hline $20,001-40,000$ & 0.00459 & 0.00589 & 0.00795 & 0.01265 & 0.02320 \\
\hline $40,001-60,000$ & 0.00219 & 0.00285 & 0.00372 & 0.00699 & 0.01980 \\
\hline
\end{tabular}

where $\pi_{\mathrm{j}}{ }^{*}$ is the known allelic frequency of allele $\mathrm{j}, \mathrm{n}$ is the number of alleles for the specific loci, $\mathbf{y}$ is the vector of offspring's genotypes, and $\alpha_{\mathrm{jk}}, \alpha_{\mathrm{sjk}}$, and $\alpha_{\mathrm{djk}}$ are the overall and sire- and dam-specific TRD parameters for the specific heterozygous pairwise combination of alleles $\mathrm{j}$ and $\mathrm{k}$, respectively.

Table A1. Threshold for the approximate empirical null distribution of transmission ratio distortion (TRD) for different ranges of numbers of informative offspring

${ }^{1}$ Informative offspring: offspring from one or both heterozygous parents for the overall TRD and from the specific heterozygous parent for the parent-specific TRD.

${ }^{2}$ Symmetric distribution with 2 tails; the threshold corresponds to the sum of both sides. 